\title{
GESTIÓN DE RESIDUOS SÓLIDOS BIODEGRADABLES PARA EL LOGRO DE LA ECOEFICIENCIA EN LA UNIVERSIDAD
}

\author{
BIODEGRADABLE SOLID WASTE MANAGEMENT AT UNIVERSITY TO \\ ACHIEVE ECOEFFICIENCY
}

Yolanda Bustamante Sánchez*

yobusa22@yahoo.es

[RECEPCIÓN: JULIO DEL 2014/CONFORMIDAD: SETIEMBRE DEL 2014]

\section{RESUMEN}

Lima produce más de dos millones de toneladas de basura al año (2'123,016 toneladas de residuos sólidos) según un informe emitido por el Ministerio del Ambiente (MINAM). En promedio, cada peruano produce $0.61 \mathrm{~kg}$ de residuos sólidos al día.

Los residuos sólidos orgánicos urbanos, RSOU, constituyen cerca del $70 \%$ del volumen total de desechos generados, que ocasionan impactos ambientales negativos a la población, medio ambiente y a la economía del país.

Se precisa la gestión integral de los RSOU y sus procesos desde la UNIVERSIDAD, que van desde la separación en la fuente (orgánico, reciclaje e inservible) hasta la transformación por el compostaje como biofertilizante y acondicionadores de suelo para la mejora de áreas verdes, aire limpio y la mejora de calidad de vida y la Ecoeficiencia en el servicio educativo que prestan las universidades y el cumplimiento de su rol en formación, investigación y proyección social.

Palabras claves: Gestión, residuos sólidos biodegradables, ecoeficiencia.

\begin{abstract}
Lima produces over 2 million tons of waste per year (2'123,016 tons of solid waste per year according to a report issued by the Ministry of Environment (MINAM). On average, every Peruvian produces $0.61 \mathrm{~kg}$ of solid waste per day.

Urban organic solid waste, RSOU constitute about $70 \%$ of the total volume of waste generated, causing negative environmental impacts on population, environment and economy.

The integrated management of RSOU and processes required from the UNIVERSITY ranging from source separation (organic, recycling and useless) to processing by composting as bio fertilizer and soil conditioner for improving green areas, air clean and improving quality of life and efficiency Eco educational service provided by the universities and the fulfillment of its role in education, research and outreach.
\end{abstract}

Keywords: Solid waste management, biodegradable, eco efficiency.

\footnotetext{
* Doctorado en Medio Ambiente y Desarrollo Sostenible (en curso) UNFV. Egresada Maestría Administración-Recursos Humanos (UNMSM) Licenciada en Educación. Posgrado Especialización en Reducción Riesgo de Desastres y Desarrollo Local Sostenible DELNET/CIF-OIT/ ONU -Turín, Italia (2011). Design, Deliver and Evaluate Training Programmes ITCILO-OIT Italia 2010. La Sostenibilidad Local: Urbanismo y Medioambiente-UIM-AECI, España 2007.
} 


\section{INTRODUCCIÓN}

Todas las sociedades humanas han tenido que enfrentar el problema de la generación de residuos sólidos. En las sociedades antiguas, la disposición de los residuos humanos y otros desechos no representaban un problema porque eran poblaciones pequeñas y la asimilación de residuos era grande. Estas practicaban formas de reciclado de residuos que usaban para fertilizar los campos de cultivo. Los problemas se inician cuando las sociedades humanas conforman las ciudades medievales, que arrojaban la basura domiciliaria a la calle, en unos zanjones centrales que, con el agua de lluvia y otras aguas servidas, producían gran pestilencia en las ciudades y la proliferación de roedores, insectos y enfermedades como la peste negra, que mató a más de la mitad de europeos del siglo XIV. En el siglo XIX, Luis Pasteur aplicó medidas sanitarias. El problema se agrava durante la Revolución industrial, a partir de la cual la contaminación ambiental se ha ido agudizando.

En la actualidad, los residuos sólidos urbanos representan un reto para instituciones como la UNIVERSIDAD, cuyo rol es vital en la gestión de estos residuos para el cumplimiento de sus objetivos de formación, investigación, proyección social y el logro de la ecoeficiencia.

El presente trabajo de investigación trata de la problemática que vivimos por la generación de residuos sólidos urbanos y cómo gestionarlos en su manejo para disminuirlos a partir de la aplicación del proceso administrativo.

\section{PLANTEAMIENTO DEL PROBLEMA}

La cantidad de residuos sólidos orgánicos y su disposición indiscriminada en rellenos sanitarios se traduce en pérdida de nutrientes y contaminación ambiental. Esta, por las características fisicoquímicas de los residuos sólidos urbanos orgánicos y los procesos de descomposición que le son propios, se corresponde con la generación de gases y lixiviados con altas cargas contaminantes, creando la necesidad de sistemas de tratamiento costosos y complejos.

El no aprovechamiento de los RSUO implica que la vida útil de los rellenos sanitarios se agote de manera más rápida. Se sabe que estos sitios escasean y además el montaje y operación de estas infraestructuras conduce a graves conflictos ambientales y sociales.
En el contexto urbano, entre los años 1940 y 2004, la población del área metropolitana de Lima y Callao se ha multiplicado en más de 12 veces, al pasar de 662 mil habitantes a más de 8 millones habitantes. Actualmente, la ciudad sigue creciendo con una tasa anual de $2.1 \%$. Se estima que en los últimos 5 años, la ciudad incorporó anualmente aproximadamente 137,000 nuevos habitantes. Lima y Callao reúnen el $29 \%$ de la población del país.

\section{OBJETIVOS}

- Aplicar las 3Rs.

- Disminuir la contaminación.

- Cuidar el medio ambiente para una mejor calidad de vida a través de la participación de la universidad.

- Lograr la ecoeficiencia en la universidad.

\section{JUSTIFICACIÓN}

La composición física de los residuos sólidos urbanos está constituida en más del $50 \%$ por residuos ORGÁNICOS, por lo que con el aprovechamiento de estos se disminuirá en gran medida la presión sobre el medio ambiente como soporte de actividades antrópicas, se reincorporarán los nutrientes al ciclo de fertilización del suelo y se frenará el uso de agroquímicos.

Una eficiente gestión integral de residuos sólidos en la universidad, desde la presentación hasta la disposición final, permitirá la aplicación de los principios de EFICIENCIA, EFICACIA Y EFECTIVIDAD en la relación costo-beneficio óptimo para el logro de la sostenibilidad ambiental.

En la legislación peruana existe una clasificación de los residuos sólidos según Ley $\mathrm{N}^{\circ} 27314$ (2000). Entre los más comunes están los residuos sólidos domiciliarios, comerciales e industriales.

El tratamiento de la basura en Lima es muy deficiente, según artículo del 31 agosto 2009 de Perú 21. La Defensoría del Pueblo advierte que municipios no cuentan con sistema de reciclaje. Indica que las alcaldías no tienen un plan integral para clasificar los desechos tóxicos en orgánicos e inorgánicos. En muchas zonas de Lima no se hace un RECOJO adecuado de la basura. En algunos distritos se pueden notar cerros de desperdicios acumulados por días. 


\section{HIPÓTESIS}

Si se gestionan los residuos sólidos orgánicos biodegradables generados en la universidad, entonces se conseguirá la ECOEFICIENCIA.

\section{METAS ESPECÍFICAS}

- Disminución de desechos $95 \%$.

- Disminución de contaminación 90 \%.

- Logro de ecoeficiencia en la universidad $95 \%$.

\section{MARCO TEÓRICO \\ RESIDUO}

Residuo es cualquier material que resulta de un proceso de fabricación, transformación, uso, consumo o limpieza, y su propietario lo destina al abandono. Los residuos sólidos se clasifican, según su origen, en residuos sólidos urbanos, residuos industriales, residuos rurales, residuos sanitarios.

\section{CARACTERÍSTICAS BIOLÓGICAS DE LOS RSU}

Casi todos sus componentes orgánicos pueden ser convertidos en gases y sólidos orgánicos e inorgánicos inertes que se integran perfectamente en el medio natural. Todos los procesos de recuperación de la materia orgánica buscan la producción de fertilizantes y el aprovechamiento del biogás. Para ello, se deben conocer las características de biodegradabilidad de la fracción orgánica de los residuos sólidos urbanos.

\section{RESIDUOS SÓLIDOS BIODEGRADABLES}

Son sustancias que pueden descomponerse en los elementos químicos que las conforman, debido a la acción de agentes biológicos, microorganismos y hongos bajo condiciones ambientales naturales.

\section{COMPOSTAJE}

Como dice Arroyave, el compostaje es un proceso natural y biooxidativo, en el que intervienen numerosos y variados microorganismos aerobios que requieren una humedad adecuada y sustratos orgánicos heterogéneos en estado sólido. Implica el paso por una etapa termófila, dando al final, como producto de los procesos de degradación de dióxido de carbono, agua y minerales como también una materia orgánica estable, libre de patógenos y disponible para ser utilizada en la agricultura como abono acondicionador de suelos, sin que cause fenómenos adversos.

\section{GESTIÓN DE RSU}

1. GENERACIÓN DE RESIDUOS: Es en la actualidad una actividad que no es muy controlable.

2. MANIPULEO Y SEPARACIÓN, ALMACENAJE Y PROCESO EN FUENTE: eL manipuleo y separación involucra las actividades asociadas con el manejo de desechos hasta que son ubicados en contenedores de recolección. El manipuleo también abarca movimiento de contenedores cargados al punto de recolección.

El almacenamiento en el sitio de generación es muy importante debido a asuntos de salud pública y consideraciones estéticas.

El procesado en FUENTE involucra actividades como la compactación, la incineración y COMPOSTADO DE DESECHOS ORGÁNICOS.

3. RECOLECCIÓN: Incluye no solo la recolección de los residuos sólidos y materiales reciclables, sino el transporte de estos materiales a lugares donde los vehículos son vaciados. Estos lugares pueden ser instalaciones de proceso de materiales, estaciones de transferencia o sitio de disposición final.

4. SEPARACIÓN, PROCESADO Y TRANSFORMACIÓN: Los medios e instalaciones para la recuperación de materiales residuales en la fuente incluyen recolectores de las veredas, volquetes y centros de recompra. La separación y el procesado se realiza en instalaciones de recuperación, estaciones de transferencia, instalaciones de incineración y sitio de disposición. El procesado incluye la separación de elementos voluminosos, separación de los componentes por tamaño mediante tamices, separación manual por rubro, reducción de tamaño por desmenuzado, separación de metales ferrosos usando imanes, reducción de volumen por compactación y combustión.

5. TRANSFERENCIA Y TRANSPORTE: Esta actividad funcional involucra dos pasos:

a. La transferencia de un vehículo pequeño de recolección a un equipamiento de mayor carga, lo que se produce en las grandes ciudades.

b. Transporte del residuos de largas distancias a centros de procesado o disposición. 
6. DISPOSICIÓN: La disposición de los residuos en los rellenos de tierra (landfills) o la diseminación en terrenos es el destino final de la mayoría de los desechos sólidos. Un LANDFILL sanitario moderno no es un vaciadero, sino una instalación ingenieril utilizada para disponer los residuos sólidos sobre la tierra o dentro de capas de tierra sin crear molestias, peligros para la salud pública o problemas de seguridad, tales como roedores e insectos y contaminación de aguas.

\section{GESTIÓN INTEGRAL DE LOS RESIDUOS SÓLIDOS}

La gestión integral de los residuos sólidos (GIRS) es la selección y aplicación de técnicas, tecnologías y programas de gestión idóneos para lograr las metas y objetivos específicos de gestión de residuos.

\section{FACTORES BÁSICOS PARA LA DETERMINACIÓN DE UN SISTEMA DE GESTIÓN DE RSU:}

1. Técnicos: Tasa de generación de RSU, composición de los RSU, existencia y/o posible desarrollo de mercados, capacidad tecnológica disponible.

2. Sociales y ambientales: Particularidades de la comunidad y la región (disponibilidad de tierras, recursos humanos), potencial impacto socioeconómico, impacto ambiental de la gestión.

\section{JERARQUÍAS EN LA GESTIÓN DE RSU}

La aplicación de una estrategia jerárquica, la cual señala la siguiente prioridad: evitar, minimizar, tratar y disponer. Significa, desde el punto de vista ambiental, que la mejor alternativa es PREVENIR, evitando la generación de un residuo. $\mathrm{Si}$ no es posible PREVENIR, se debe buscar su MINIMIZACIÓN (las 3R, reducir en cantidad y/o peligrosidad, reusar y reciclar aprovechando los materiales y/o energía que contienen el residuo). En tercer término si no es posible MINIMIZAR, se debe buscar su TRATAMIENTO, con el objetivo de reducir cantidad y/o peligrosidad antes de la DISPOSICIÓN FINAL del residuo.

Las prioridades para la jerarquización de las acciones propuestas por la Agencia de Protección Ambiental de los Estados Unidos (EPA) pueden ser adoptadas por los países desarrollados. Sin embargo, para los países en vías de desarrollo se requieren algunas modificaciones. Como sigue:
1. Prevención: La reducción de peso y volumen en origen y la reutilización son los pilares fundamentales de la prevención.

2. Reducción en origen: Puede realizarse durante las etapas de diseño, fabricación y embalaje de los productos

3. Recuperación: Reúso y reciclado. Es la segunda prioridad que implica la separación y la recolección de material secundario, la clasificación, el acopio y la preparación para el reúso, reciclaje y transformación en nuevos productos.

La recuperación es un factor importante para ayudar a reducir la demanda de recursos y energía, a su vez que se reduce la cantidad de residuos que requieren ser dispuestos en un relleno sanitario

4. Transformación y tratamiento: Implica la alteración fisicoquímica o biológica de los residuos. El propósito es mejorar la EFICACIA de las operaciones y sistemas de GESTIÓN DE RESIDUOS, recuperar materiales, conversión en energía en forma de calor, obtención de biogás como combustible o humus (compost) como mejorador del suelo.

5. Eliminación final: Debe llevarse a cabo en todos los residuos que no puedan ser evitados, reciclados o valorizados. La disposición en relleno sanitario está en la última posición o escala de valores porque representa la forma menos deseada de tratar a los residuos, sin embargo hasta ahora es inevitable. Cualquiera que sea el sistema de tratamiento de los RSU siempre conlleva la existencia de un relleno sanitario como solución complementaria a su funcionamiento.

- Entre los beneficios que trae aparejado se pueden tener en cuenta el control de la polución de aguas, la reducción de la emisión de olores y la reducción del impacto visual, a su vez se produce biogás fácilmente recuperable.

\section{CRITERIOS A TENER EN CUENTA EN UN SISTEMA DE GESTIÓN DE RSU}

Por lo anterior, todo proyecto de residuos sólidos debe contemplar cinco componentes básicos:

- Aspectos técnicos: Se refieren al análisis de las operaciones tecnológicas posibles y apropiadas para solucionar el problema. Su alcance incluye consideraciones técnicas, estudios y presupuestos de obras 
- Aspectos económicos: Implica que la alternativa seleccionada deberá someterse a un análisis económico.

- Aspectos financieros.

- Aspectos institucionales: El proyecto debe demostrar la capacidad administrativa y de gestión, tanto de organismo ejecutor de las obras como del administrador del servicio, con el fin de hacer posible su realización y operación eficiente.

- Aspectos sociales y ambientales: Se consultarán las poblaciones afectadas, al igual que la voluntad y capacidad de pago de los usuarios $\mathrm{y}$, en el caso que amerite, se analizarán los posible impactos ambientales derivados de la ejecución y funcionamiento del proyecto, con el fin de prever acciones destinadas a contrarrestar los efectos adversos.

\section{GESTIÓN INTEGRAL DE RSU}

1. La gestión integral de residuos sólidos, GIRSU, es la disciplina asociada al control de la GENERACIÓN, ALMACENAMIENTO, RECOLECCIÓN, TRANSFERENCIA Y TRANSPORTE, TRATAMIENTO Y DISPOSICIÓN FINAL de estos. Incluye el seguimiento administrativo, de forma que armonice con los principios de la salud pública, de la economía, de la ingeniería, de la conservación, de la estética y de otras consideraciones ambientales y que también responda a las expectativas de la opinión pública.

2. La manipulación de residuos y separación, almacenamiento y procesamiento en el origen: el segundo de los 06 elementos funcionales en el sistema de gestión de los residuos sólidos es la manipulación de residuos, la separación, el almacenamiento y el procesamiento en origen o prerecogida. La manipulación y la separación de residuos comprenden todas aquellas operaciones que se llevan a cabo en referencia a los residuos sólidos urbanos y hasta su ubicación en los lugares de almacenamiento para su recogida. Pueden distinguirse dos tipos de pre-recogida.

- Prerrecogida sin selección: En este caso no se lleva a cabo ninguna manipulación ni separación de los residuos sólidos urbanos en origen por parte del ciudadano.

- Prerrecogida con selección: Con este tipo de re-recogida se separan las fracciones en origen.
3. Recogida: Incluye tanto elementos sólidos como reciclables. Dentro de la gestión de residuos sólidos urbanos, la recogida constituye la fase de mayor envergadura que puede llegar a representar entre el $70 \%$ y el $90 \%$ de los costes del servicio.

4. Separación, procesamiento y transformacion de residuos sólidos.

5. Ecoeficiencia.

Una definición de ecoeficiencia es la siguiente: Proporcionar bienes y servicios a un precio competitivo, que satisfaga las necesidades humanas y la calidad de vida, al tiempo que reduzca progresivamente el impacto ambiental y la intensidad de la utilización de recursos a lo largo del CICLO DE VIDA, hasta un nivel compatible con la capacidad de carga estimada del planeta (World Business Council for Sustainable Development (WBCSD).

\section{MARCO NORMATIVO}

- Declaración Talloires.

- Conferencia Mundial sobre Educación Ambiental en Tbilisi (Rusia) 1977.

- Conferencia Mundial sobre Educación y Formación Ambiental UNESCO /PNUMA. Moscú 1987.

- LEY DE RESIDUOS SOLIDOS NRO 27314 y Ley General del Ambiente Nro. 28611.

\section{MÉTODO}

Para el logro de estos objetivos se requiere la aplicación del PROCESO ADMINISTRATIVO (planeamiento, organización, dirección y control) y sus estrategias para el fortalecimiento de capacidades, la gestión de la información, el establecimiento de programas de investigación, el desarrollo de la tecnología para la proyección social a través de un trabajo en equipo en la universidad para el logro de la ECOEFICIENCIA.

\section{RESULTADOS}

- Menos contaminación del aire.

- Mejor calidad de vida en la comunidad universitaria.

- Cultura ambiental, según plan de educación progresiva y trabajo participativo.

- Universidad activa con proyección social. 


\section{DISCUSIÓN}

- La gestión de residuos sólidos biodegradables para el logro de la ecoeficiencia en la universidad conlleva trabajo lento, perseverante, creativo, motivador, que implica compromiso de todos los participantes

- Se aprecia el rol significativo de la universidad como mediador del conocimiento y la aplicación de este, lo que constituye un aporte trascendente para el desarrollo local.

- La investigación genera apoyo de las autoridades hacia los investigadores, con recursos de diversa índole para el logro de las metas a alcanzar y la satisfacción de la sociedad y el desarrollo local.

\section{CONCLUSIONES}

- Los residuos sólidos urbanos, parte de los recursos finitos del planeta que causan enfermedades y daños ambientales (planificación en RSU).

- El aprovechamiento de los RSOU no se debe ver como un proceso aislado; debe ser integrado con las empresas que prestan el servicio público del aseo, generando más cultura y convicción del usuario con relación al ambiente (Organización para reducir contaminación).

- Estimular la compensación a los entes o personas que contribuyan con el mejoramiento del ambiente, aprovechando los residuos, debido a que minimiza la disposición en el relleno sanitario y aumenta su vida útil (dirección, organización y 3R (reduce, reúsa y reutiliza).

- Revisar la normatividad y su cumplimiento en las instituciones que deben presentar planificación ambiental, sus planes operativos, financieros, ambientales (planificación y 3R).

- Capacitar a recursos humanos de las instituciones públicas, privadas, la comunidad (dirección para calidad del medio ambiente).

- La actividad primordial para el aprovechamiento de los residuos sólidos orgánicos urbanos es la SEPARACIÓN EN LA FUENTE (dirección, capacitación en RSU).

- Las políticas para reducir la generación de residuos municipales aún no han dado resultados significativos (planificación ambiental)
- La aplicación de las TECNOLOGÍAS para el aprovechamiento de los RSOU deben ser promovidas (dirección, formación, TIC y medio ambiente).

- El aprovechamiento de los residuos sólidos urbanos es una actividad deseable desde el punto de vista ambiental. Las normas deben indicar el carácter de OBLIGATORIO, el municipio debe promover la viabilidad de proyectos de aprovechamiento sostenibles económica y financieramente (organización, dirección y ecoeficiencia)

- Existe la burocracia e intereses políticos en la organización interna de los municipios, ministerios e instituciones públicas a cargo del manejo ambiental (organización, control y reduce la contaminación)

- Falta de educación comunal, capacitación interna de autoridades, mejora de tecnologías (dirección, formación, TIC para aplicación de $3 R$ : reduce, reúsa y reutiliza)

\section{REFERENCIAS BIBLIOGRÁFICAS}

- González Lopez, Antonio (2002). "La preocupación por la calidad del medio ambiente. Un modelo cognitivo sobre la conducta ecológica“. Universidad Complutense de Madrid. Madrid 2002.

- Rendón Gutiérrez, Rosa Elba (2010). “Espacios Verdes públicos y calidad de vida“. . Universidad de Guadalajara, México.

- Bolaños, Karla (2012). . Cuarto Informe Nacional de Residuos Sólidos Municipales y no Municipales". Ministerio del Ambiente. Setiembre 2012.

- Tavera Cortes, María Elena (2009). “Proyecto de viabilidad tecnológica para producción de composta mediante residuos orgánicos" Instituto Politécnico Nacional.

- Sorensen, MARK, Barzetti Valerie (1998). " Manejo de las áreas Verdes". Washington D.C. MAYO 1998.

- Wbcsd, Ocde (1992). Programa sobre la Ecoeficiencia, experiencias Ministerio de Medio Ambiente Australiano. Eco efficiency Case Study Collection, Sustainable Business Challenge (SBC). 
PROYECTO DE PRODUCCIÓN DE COMPOST

\begin{tabular}{|c|c|c|c|c|c|c|c|c|c|c|}
\hline \multirow[b]{2}{*}{ TIPO DE RESIDUO } & \multicolumn{7}{|c|}{ RECOLECCIÓN DE RESIDUOS POR DÍA (DATOS EN KILOS) } & \multirow[b]{2}{*}{$\begin{array}{c}\text { SUB- } \\
\text { TOTAL } \\
\end{array}$} & \multirow[b]{2}{*}{ PROMEDOO } & \multirow[b]{2}{*}{$\begin{array}{l}\text { VALORES } \\
\text { EN } \\
\text { PORCENT. }\end{array}$} \\
\hline & DiA 1 & DiA 2 & DiA 3 & DiA 4 & DiA 5 & DiA 6 & DiA 7 & & & \\
\hline MATERLA ORGÁNICA & 100,35 & 90,5 & 110 & 95 & 90 & 100,2 & 119,45 & 706 & 100,97 & $58.84 \%$ \\
\hline PAPEL Y PLASTIOOS & 15,12 & 13 & 16.5 & 14,25 & 13.5 & 15,03 & 32,63 & 120,03 & 17,15 & $10,00 \%$ \\
\hline LIQUIDOS PRODUCIDOS & 514 & 51,4 & 51.4 & 51.4 & 51.4 & 51.4 & 514 & 359.8 & 51,40 & $29,99 \%$ \\
\hline ETIERCOL & 2 & 2 & 2 & 2 & 2 & 2 & 2 & 14 & 2,00 & $1,17 \%$ \\
\hline TOTAL & 169.37 & 156,9 & 179,9 & 162,55 & 156,9 & $16,8,5$ & $205,4 \mathrm{~B}$ & 1199,83 & & $100,00 \%$ \\
\hline
\end{tabular}

Fuente: Elaboración propia

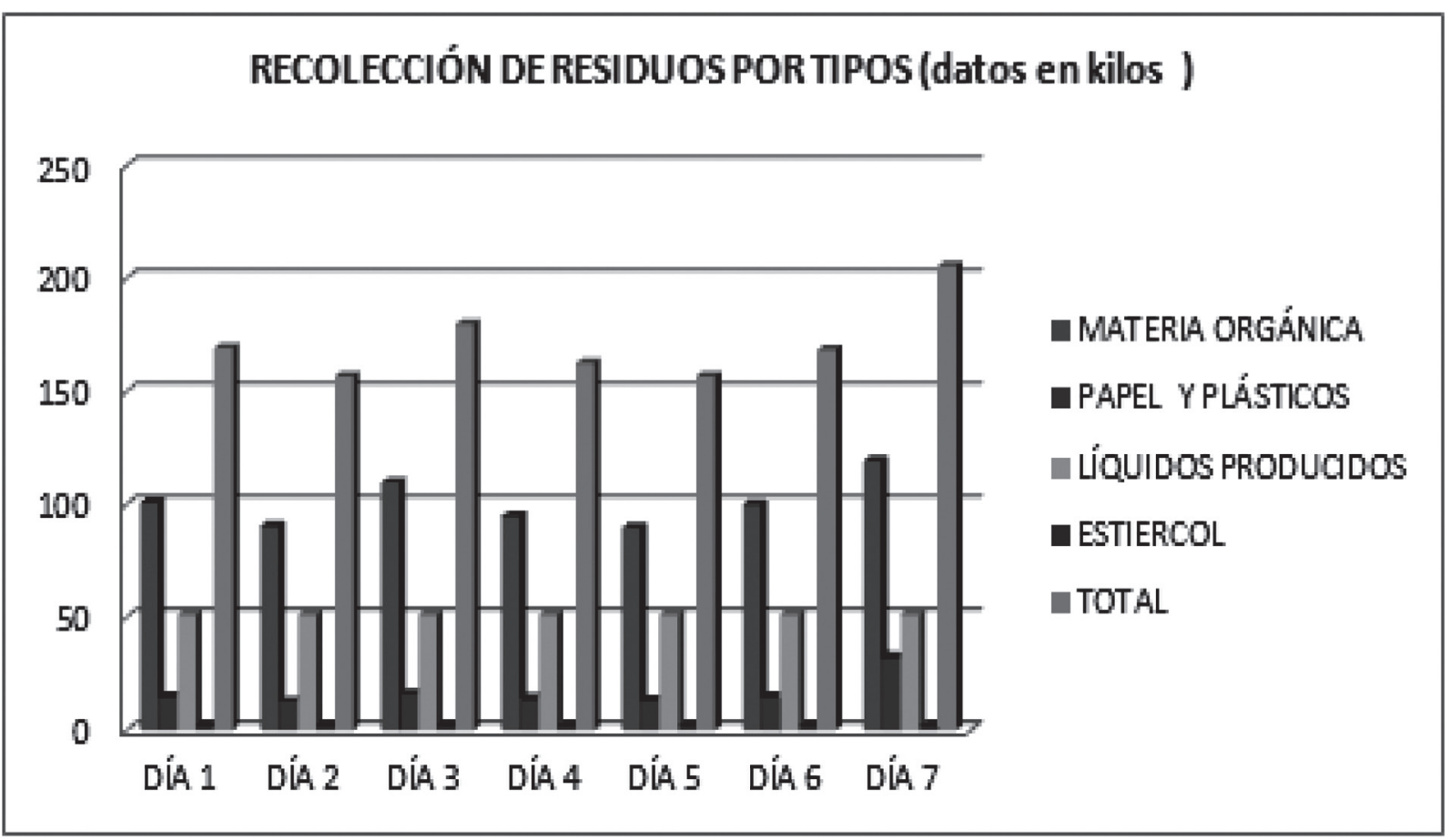

Fuente: Elaboración propia 